\title{
Radiative cascades in charged Quantum Dots
}

\author{
E Poem, Y Kodriano, C Tradonsky and D Gershoni \\ Department of physics, The Technion - Israel institute of technology, Haifa, 32000, Israel
}

\section{B D Gerardot and P M Petroff}

Materials Department, University of California Santa Barbara, CA, 93106, USA

E-mail: poem@technion.ac.il

\begin{abstract}
We measured, for the first time, two photon radiative cascades due to sequential recombination of quantum dot confined electron hole pairs in the presence of an additional spectator charge carrier. We identified direct, all optical cascades involving spin blockaded intermediate states, and indirect cascades, in which non radiative relaxation precedes the second recombination. Our measurements provide also spin dephasing rates of confined carriers.
\end{abstract}

Semiconductor quantum dots (QDs) strongly localize charge carriers, and discretize their energy level spectrum, in a similar way to electrons in atoms. Radiative cascades in neutral QDs [1, 2, 3, 4] demonstrated their potential as deterministic sources for polarization entangled photon pairs [5, 6]. The neutral radiative cascade [7, 8] leaves the QD empty of charge carriers. This is essential for entangling the emitted two photons, since otherwise the remaining carrier's spin betrays the required "which path" ambiguity [9, 10]. Neutrality prevents, however, the important benefit of correlating between the emitted photons' polarizations ("flying qubits") and the local carrier's spins ("anchored qubits"). The situation is drastically different in charged QDs, where quantum correlations exist between the flying and anchored qubits. Here we report on two-photon radiative cascades in the presence of an additional hole.

The energy levels of a positively charged QD [11, 12] containing up to three holes and two electrons are schematically described in Fig. 1(a). The figure presents also the relevant optical and non-optical total-spin conserving transitions between these levels. The two photon radiative cascades start from the ground level of the three hole and two electron state. The unpaired hole's spin projection along the growth axis determines the total spin of the two Kramers' degenerate states (for simplicity only one state is drawn in Fig. 1(a)). Radiative recombination of first level electron-hole (e-h) pair leaves three unpaired charge carriers within the QD. There are 8 possible different spin configurations for the remaining carriers. These configurations form 4 energy levels of Kramers' pairs [11, 12. The three lowest levels are those in which the two unpaired holes are in spin-triplet states. Those states are separated from the highest energy level in which the holes are in a singlet spin state by the hole-hole isotropic exchange interaction, which is significantly stronger than the e-h exchange interaction. The later removes the degeneracy between the triplet states as shown in Fig. 1(a). The lowest triplet level cannot be reached optically. The optical transitions into the other levels are optically allowed. The circular polarization of the emitted photons are indicated in the figure. It depends on the spins of the annihilated electron hole pair. The measured emission contains also linear components (see Fig. 1(c)), due to the anisotropic 
e-h exchange interaction [11, 12]. The relaxation proceeds by radiative recombination of the remaining first level e-h pair, leaving thus only one hole in its second level. The hole can then quickly relax non-radiatively to its ground level. There is a fundamental difference between the singlet and triplet intermediate states. While in the later, due to Pauli's exclusion principle, radiative recombination must occur before the excited hole can relax to its ground state (resulting in two "direct" cascades), in the former non-radiative relaxation of the excited hole state may occur prior to the radiative recombination (resulting in one "direct" and one "indirect" cascade).

Figure 1. (a) Schematic description of the energy levels of a singly positively charged QD. Vertical (curly) arrows indicate radiative (nonradiative) transitions between these levels. State occupation and spin wavefunctions are described to the left of each level where $\uparrow(\Downarrow)$ represents an electron (hole) with spin up (down). A short blue (long red) arrow represents a carrier in its first (second) level. S (T) stands for two holes' singlet (triplet) state and 0 (1) for $S_{z}=0$ $\left(S_{z}= \pm 1\right)$ total holes' pseudo-spin projection on the QD growth direction. The ground staste singlet is indicated by $\mathrm{S}_{G}$. Only one out of two (Kramers') degenerate states is described. (b) Measured PL spectrum

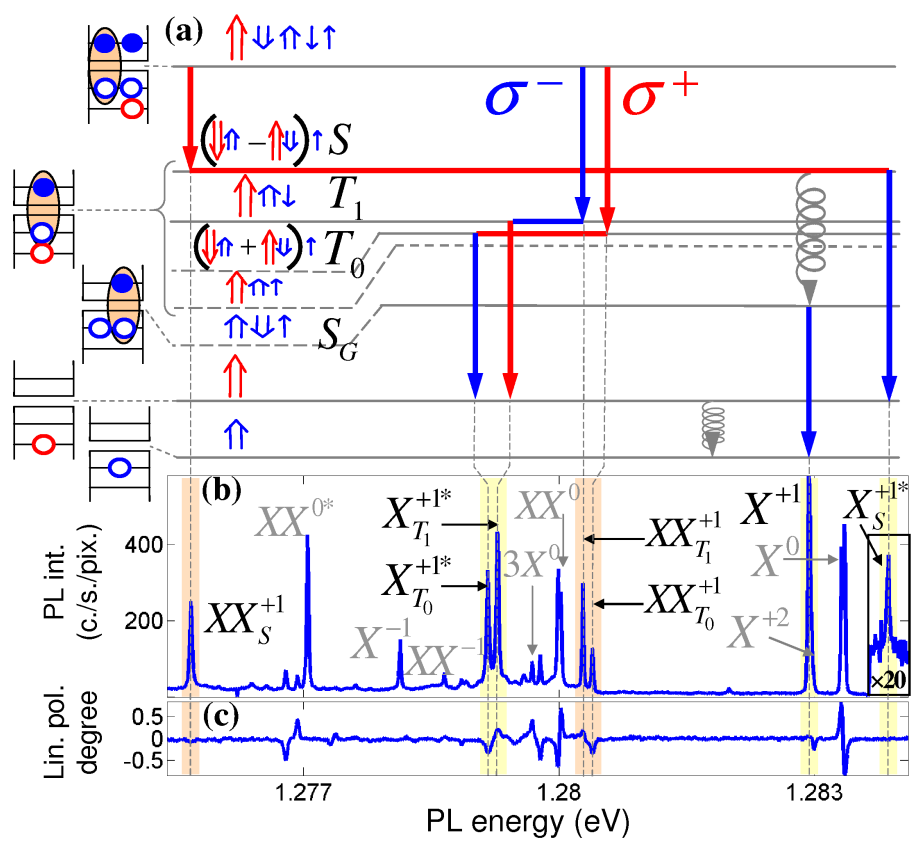
on which the actual transitions are identified. Excitonic (biexcitonic) transitions are highlighted yellow (orange). Transitions which are not discussed here are marked by gray letters. (c) Measured degree of linear polarization spectrum, along the in-plane symmetry axes of the QD. Positive (negative) value represents polarization along the QD's major (minor) axis.

The studied sample contains InGaAs QDs in the middle of a $1 \lambda$ microcavity [5]. For the optical measurements the sample was placed inside a tube immersed in liquid Helium, maintaining sample temperature of $4.2 \mathrm{~K}$. A X60, $0.85 \mathrm{NA}$, in-situ microscope objective was used to both focus the exciting beam on the sample surface and to collect the emitted light. The polarization of the emitted light was analyzed using two computer controlled liquid crystal variable retarders and a linear polarizer. In Fig. 1(b) we present the spectrum measured under non-resonant $\mathrm{cw}$ excitation with $1 \mu \mathrm{W}$ of HeNe laser light $(1.96 \mathrm{eV})$. The corresponding degree of linear polarization is presented in Fig. 1(c). The spectral lines participating in the radiative cascades described in Fig. 1(a) are clearly identified spectrally in the single QD PL and linear polarization spectra, and are highlighted orange (yellow) for biexcitonic (excitonic) transitions. For polarization-sensitive time-resolved intensity-correlation measurements, we used a HanburyBrown and Twiss like apparatus [5]. In Fig. 2 we present the measured and calculated intensity correlation functions for photon pairs emitted in the four spin-conserving radiative cascades outlined in Fig. 1(a). The measured data clearly reveal the sequence of the radiative events, reassuring the interpretations of Fig. 1. In Fig. 3 we present measured and calculated intensity correlation functions between different radiative cascades. Since spin blockading prevents the relaxation of the second level hole to its first level, they provide an estimate for the rate by which 
the hole spin's scatters [13. Fast scattering would give rise to a peak in the correlation function, because then the photon emissions preceeding and succeeding the scattering process would mostly happen one right after the other. Scattering rate slower than the radiative recombination rate and/or the optical generation rate would give rise to a dip in the correlation function, since the second photon would most probably be emitted only after additional recombination and generation of e-h pairs. In Fig. 3 (a) and (c) we probe possible transitions from the singlet

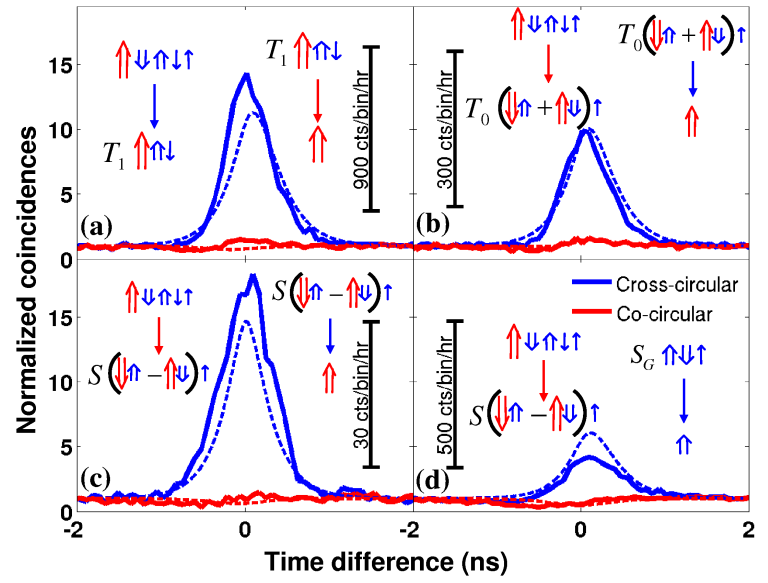

Figure 2. Measured and calculated timeresolved, polarization sensitive intensity correlation functions, for the 4 radiative cascades described in Fig. 1, The states involved in the first (second) photon emission are illustrated to the left (right) side of each panel. All symbols and labels are as in Fig. 1. Solid Blue (red) line stands for measured cross- (co-) circularly polarized photons. Dashed lines represent the corresponding calculated functions. The bar presents the acquisition rate in coincidences per time bin (80 ps) per hour.

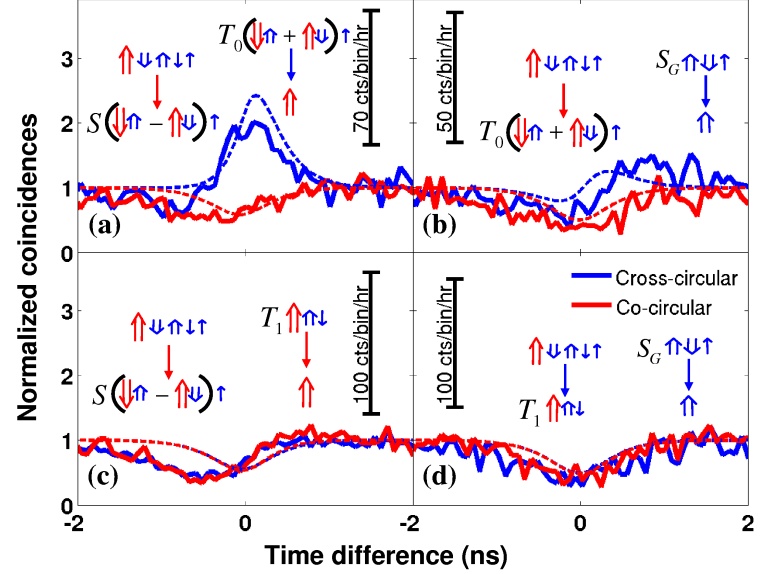

Figure 3. Measured and calculated timeresolved, polarization sensitive intensity correlation functions, across the radiative cascades. (a) [(c)] Correlations between the singlet biexciton transition and the exciton transition from the $T_{0},\left[T_{1}\right]$ state. (b) $[(\mathrm{d})]$ Correlations between the $T_{0},\left[T_{1}\right]$ biexciton transition and the ground $X^{+1}$ exciton transition. All symbols and labels are as in Fig. 1. The meanings of all line types and colors are as in Fig. 2 .

intermediate state to the triplet $T_{0}$ and $T_{1}$ intermediate states, respectively. In (b) and (d) we probe possible transitions from the triplet $T_{0}$ and $T_{1}$ intermediate states, respectively, to the singlet ground state. Assuming that relaxation from the intermediate triplet states to the ground singlet states must be preceded by transition to the intermediate singlet states, these measurements provide quantitative estimation for the reverse of the processes described in (a) and (c). From the measured data in Fig. 3 one clearly notes that transition between the two holes' singlet state to the $T_{1}$ triplet state (Fig. 3r) and vice versa (Fig. 3 d ) are forbidden, while transitions between the singlet and the $T_{0}$ triplet states (Fig. $3 \mathrm{a}$ ) and vice versa (Fig. $3 \mathrm{~b}$ ) are partially allowed. This means that the holes spin projection on the QD's growth axis is conserved during the relaxation while their in-plane spin projection scatters [13]. The difference between the scattering rates from the singlet to triplet state and that from the triplet to singlet is due to the energy difference between these two states $(\sim 4 \mathrm{meV})$, which is much larger than the ambient thermal energy $(\sim 0.5 \mathrm{meV})$.

Our model is composed of a set of coupled rate equations for the time-dependent probabilities of finding the system in one of its many-carriers-states [2. We include all the states as described in Fig. 1(a), together with their Kramers conjugates. In addition, we include four more states 
representing charged multiexcitons up to 6 e-h pairs 2]. There are clear spectral evidences for processes in which the QD changes its charge state and becomes neutral due to optical depletion [14, 15, 16] (see Fig. 1(b)). These observations are considered in our model by introducing one additional state which represents a neutral QD. The transition rates between the states include radiative rate $\left(\gamma_{r}=1.25 n s^{-1}\right.$ deduced directly from the PL decay of the exciton lines) and non-radiative spin-conserving rate $\left(\Gamma_{S \rightarrow S}=35 \gamma_{r}\right.$, deduced from the intensity ratios of the relevant PL lines). We also include the rates for optical generation of e-h pairs $\left(G_{e}=1 \gamma_{r}\right.$, forced by equating between the emission intensities of the biexciton and exciton spectral lines), optical depletion, and recharging $\left(G_{D}=4 \gamma_{r}\right.$ and $G_{C}=0.1 \gamma_{r}$ as deduced from the relevant line intensity ratios, and correlation measurements between the neutral and charged exciton).

The data clearly show that hole spin scattering rates, $\left(\Gamma_{S \leftrightarrow T_{1}}\right)$ which do not conserve the spin projection on the QD's growth axis, are vanishingly small. Therefore we set them to 0 . In order to account for the observed correlations between singlet $(S)$ and $T_{0}$ states, Fig. 3 , we fitted in-plane scattering rates [13] $\Gamma_{T_{0} \rightarrow S}=0.6 \gamma_{r}$ and $\Gamma_{S \rightarrow T_{0}}=10 \gamma_{r}$ (such processes still conserve the projection of the total spin along the growth axis). The ratio between these rates simply gives the temperature of the optically excited QD $(\sim 19 \mathrm{~K})$. The anisotropic e-h exchange interaction mixes the $T_{0}$ and $T_{1}$ states [12, 11]. This makes the natural polarizations of the relevant transitions elliptical rather than circular. The mixing degree is obtained from the measured degree of linear polarization of the biexciton transitions [12]. Our model considers this mixing as well. It explains the non-zero correlations in co-circular polarizations.

The 2 inplane hole's spin scattering rates that we fitted describe very well the 16 measured intensity correlation functions. The calculated functions convoluted with the system response are presented in Fig. 2 and Fig. 3 by dashed lines.

In summary, we identified 3 direct and one indirect radiative cascades in singly charged QDs and demonstrated unambiguous correlations between the polarizations of the emitted photons and the spin of the remaining charge carrier. Our correlation measurements show that while holes' spin-projection conserving scattering rates are few times faster than the radiative rates, spin-projection non-conserving rates are vanishingly small.

\section{Acknowledgments}

The support of the US-Israel binational science foundation (BSF), the Israeli science foundation (ISF), the Israeli ministry of science and technology and the Technion's RBNI are gratefully acknowledged.

\section{References}

[1] Moreau E, Robert I, Manin L, Thierry-Mieg V, Gérard J M and Abram I 2001 Phys. Rev. Lett. 87183601

[2] Regelman D V, Mizrahi U, Gershoni D, Ehrenfreund E, Schoenfeld W V and Petroff P 2001 Phys. Rev. Lett. 87257401

[3] Kiraz A, Falth S, Becher C, Gayral B, Schoenfeld W, Petroff P, Zhang L, Hu E and Imamoglu A 2002 Phys. Rev. B 65161303

[4] Santori C, Fattal D, Pelton M and Yamamoto Y 2002 Phys. Rev. B. 66045308

[5] Akopian N, Lindner N H, Poem E, Berlatzky Y, Avron J, Gershoni D, Gerardot B D and Petroff P M 2006 Phys. Rev. Lett. 96130501

[6] Hafenbrak R, Ulrich S M, Michler P, Wang L, Rastelli A and Schmidt O G 2007 New J. Phys. 9315

[7] Gammon D, Snow E S, Shanabrook B V, Katzer D S and Park D 1996 Phys. Rev. Lett. 763005

[8] Kulakovskii V D, Bacher G, Weigand R, Kümmell T and Forchel A 1999 Phys. Rev. Lett. 821780

[9] Troiani F and Tejedor C 2008 Phys. Rev. B 78155305

[10] Avron J E, Bisker G, Gershoni D, Lindner N H, Meirom E A and Warburton R J 2008 Phys. Rev. Lett. 100 120501

[11] Kavokin K V 2003 Phys. Stat. Sol. (a) 195592

[12] Akimov I A, Kavokin K V, Hundt A and Henneberger F 2005 Phys. Rev. B 71075326

[13] Poem E, Khatsevich S, Benny Y, Marderfeld I, Badolato A, Petroff P M and Gershoni D 2009 Solid State Commun. 1491493 
[14] Hartmann A, Ducommun Y, Kapon E, Hohenester U and Molinari E 2000 Phys. Rev. Lett. 845648

[15] Baier M H, Malko A, Pelucchi E, Oberli D Y and Kapon E 2006 Phys. Rev. B 73205321

[16] Ulrich S M et al. 2005 Phys. Rev. B 71235328 